\title{
Robust Passivity and Feedback Design for Nonlinear Stochastic Systems with Structural Uncertainty
}

\author{
Zhongwei Lin, Jizhen Liu, and Yuguang Niu \\ State Key Laboratory of Alternate Electrical Power System with Renewable Energy Sources, \\ School of Control and Computer Engineering, North China Electric Power University, Beijing 102206, China
}

Correspondence should be addressed to Zhongwei Lin; linzhongwei2003@tom.com

Received 30 January 2013; Accepted 18 February 2013

Academic Editor: Weihai Zhang

Copyright (C) 2013 Zhongwei Lin et al. This is an open access article distributed under the Creative Commons Attribution License, which permits unrestricted use, distribution, and reproduction in any medium, provided the original work is properly cited.

This paper discusses the robust passivity and global stabilization problems for a class of uncertain nonlinear stochastic systems with structural uncertainties. A robust version of stochastic Kalman-Yakubovitch-Popov (KYP) lemma is established, which sustains the robust passivity of the system. Moreover, a robust strongly minimum phase system is defined, based on which the uncertain nonlinear stochastic system can be feedback equivalent to a robust passive system. Following with the robust passivity theory, a global stabilizing control is designed, which guarantees that the closed-loop system is globally asymptotically stable in probability (GASP). A numerical example is presented to illustrate the effectiveness of our results.

\section{Introduction}

It is well known that passivity theory plays an important role in many engineering problems, which is a powerful technique in handling stability issue. Many problems on related topics have been investigated; see [1-4]. In 1990s, much more attention has been focused on the development of synthesis technique that combines the passivity theory with geometric nonlinear control theory; see [5-7] and the references therein. The work in [5] developed a framework for studying the stabilizability of minimum phase deterministic nonlinear systems. Especially, [8] has proposed a robust enhancement of the result in [5], which discussed the passivity and global stabilization for a class of uncertain minimum phase nonlinear systems, which considers the nonlinear systems with structural uncertainties, that is, gain-bounded uncertainties. The work in [9] extended the corresponding approach to solve the robust almost disturbance decoupling problem for such a class uncertain nonlinear systems.

On the other hand, due to the great many applications of stochastic Itô systems in real world [10], the study on feedback controller design for such a class of systems has received a great deal of attention; see [11-19] and the references therein. Especially, many researchers have extended the existing passivity theory from deterministic systems to stochastic systems. The work in [11] obtained necessary and sufficient conditions for the existence of diffeomorphisms that transform stochastic nonlinear systems to various canonical forms. The work in [12] studied the problem of feedback equivalent to a passive system for a particular class of interconnected stochastic systems. The work in [13] developed a systematic method for global asymptotic stabilization in probability of nonlinear control stochastic differential systems based on the passivity theory. However, compared with the deterministic case, up to date, there still requires much work of investigating the passivity theory of uncertain nonlinear stochastic systems with structural uncertainties.

This paper considers a class of uncertain nonlinear stochastic systems which are expressed by the Itô-type stochastic differential equations with structural uncertainty. We shall investigate the problem of feedback equivalent to a robust passive system and global stabilization for uncertain nonlinear stochastic system via robust passivity theory. A robust stochastic KYP lemma is proposed, which can be regarded as a robust stochastic extended results in deterministic case $[2,5]$. Based on the above, we investigate the relationship between a robust passive system and the corresponding zero-output dynamics, where a robust strongly minimum phase property is proposed. Then, sufficient conditions for global asymptotic stabilization in probability are provided. 
The remainder of the paper is organized as follows: Section 2 develops the robust passivity theory for a class of uncertain nonlinear stochastic systems, which presents a robust stochastic version of KYP lemma. In Section 3, through adopting the appropriate diffeomorphism, we discuss the relationship between the robust passivity of the system and the stability of the zero-output system, that is, the robust minimum phase system. Then, the global stabilizing control of the system can be determined through discussing the robust strongly minimum phase property. Based on the robust stochastic passivity theory, sufficient conditions are given for global stabilization of such a class of uncertain nonlinear stochastic systems. In Section 4, an example is given to illustrate the usefulness of our results. Section 5 concludes this paper.

For convenience, we adopt the following notations:

$\mathcal{S}_{n}$ : the set of all real $n \times n$ symmetric matrices;

$A^{\prime}$ : the transpose of a matrix or vector $A$;

$A \geq 0(A>0): A$ is a positive semidefinite (positive definite) matrix;

$\mathscr{C}^{2}(U)$ : the class of functions $V(x)$ twice continuously differentiable with respect to $x \in U$;

$L_{g} \lambda$ : the Lie derivative of a smooth function $\lambda$ along the vector field $g$, that is, $L_{g} \lambda:=(\partial \lambda / \partial x) g$;

$R^{n}: n$-dimensional Euclidean space;

$\|x\|: 2$-norm of a vector $x \in R^{n}$.

\section{Robust Stochastic Passivity Theory}

First of all, let $\left(\Omega, \mathscr{F},\left\{\mathscr{F}_{t}\right\}_{t \geq 0}, P\right)$ be a given filtered probability space where there lives a standard one-dimensional Brownian motion $w(t)$ on $[0,+\infty)$ with $w(0)=0$ and $\mathscr{F}_{t}=\sigma\{w(s) \mid$ $0 \leq s \leq t\}$. The Brownian motion is assumed to be one dimensional only for simplicity, because there is no essential difference from the multidimensional case.

Consider the following uncertain nonlinear stochastic control system governed by Itô's differential equation:

$$
\begin{gathered}
d x=(f(x)+\Delta f(x)+g(x) u) d t \\
+l(x) d w, \quad f(0)=0, l(0)=0, \\
y=h(x), \quad h(0)=0,
\end{gathered}
$$

where $x(t) \in R^{n}$ is the state vector, $x_{0} \in R^{n}$ is the initial state, and $y(t) \in R$ is the controlled output. $w(t)$ is an onedimensional Brownian motion. $u(t) \in R$ is the control input, which is an adaptive process with respect to $\left\{\mathscr{F}_{t}\right\}_{t \geq 0} . f, g$, $l$, and $h$ are assumed to be smooth functions of appropriate dimensions. $\Delta f(x): R^{n} \rightarrow R^{n}$ represents structural uncertainty or uncertain perturbation characterized by

$$
\Delta f(x)=e(x) \delta(x), \quad \Delta f(0)=0,
$$

where $e(x): R^{n} \rightarrow R^{n \times m}$, with $e(0)=0$ being a known matrix whose entries are given smooth functions, and $\delta(x)$ : $R^{n} \rightarrow R^{m}$ is an unknown vector-valued function. It is assumed that $\delta(x)$ is constrained to a given smooth function $n(x): R^{n} \rightarrow R^{m}$, that is,

$$
\Gamma=\{\delta(x):\|\delta(x)\| \leq\|n(x)\|\} .
$$

If $\delta(x) \in \Gamma$, then $\Delta f(x)$ is said to be admissible.

Definition 1. An uncertain system of the form (1) is said to be robust passive on $[s, \infty), s \geq 0$ if there exists a nonnegative continuous function $V(x): R^{n} \rightarrow R^{+}$, called the storage function, such that for all $t \geq s \geq 0, x(s) \in R^{n}$, and for all admissible $\Delta f(x)$,

$$
E V(x(t))-V(x(s)) \leq E \int_{s}^{t} y^{\prime}(\tau) u(\tau) d \tau .
$$

Here, (4) can be regarded as the robust version of the passive inequality for stochastic systems. For simplicity of our following discussion, we assume that the storage function of (4), if it exists, belongs to $\mathscr{C}^{2}\left(R^{n}\right)$.

In view of Definition 1 and [8], it is quite natural to introduce the following concept for uncertain nonlinear stochastic systems.

Definition 2. System (1) is said to have the robust KYP property if there exists a nonnegative function $V \in \mathscr{C}^{2}\left(R^{n}\right)$ : $R^{n} \rightarrow R^{+}$, with $V(0)=0$, such that

$$
\begin{gathered}
L_{f+\Delta f} V(x)+\frac{1}{2} l^{\prime} \frac{\partial^{2} V(x)}{\partial x^{2}} l \leq 0, \\
L_{g} V(x)=h^{\prime} .
\end{gathered}
$$

If the above inequality becomes a strict inequality that holds for a positive definite function $V(x)$, then system (1) is said to be robust strictly passive.

Now, we derive conditions under which an uncertain nonlinear stochastic system is robust passive, which can be viewed as a robust stochastic version of the nonlinear KYP lemma, which plays an important role in studying global robust stabilization for uncertain nonlinear stochastic systems.

Theorem 3. System (1) is robust passive if and only if there exists a nonnegative function $V \in \mathscr{C}^{2}\left(R^{n}\right): R^{n} \rightarrow R^{+}$, with $V(0)=0$, such that

$$
\begin{gathered}
L_{f} V(x)+\left\|L_{e}^{\prime} V(x)\right\|\|n(x)\|+\frac{1}{2} l^{\prime} \frac{\partial^{2} V(x)}{\partial x^{2}} l \leq 0, \\
L_{g} V(x)=h^{\prime},
\end{gathered}
$$

where $L_{e}^{\prime} V(x)=\left(L_{e} V(x)\right)^{\prime}$.

Proof. Sufficiency. According to the Cauchy inequality and considering the fact of $\|\delta(x)\| \leq\|n(x)\|, \forall \delta(x) \in \Gamma$, we have

$$
\begin{aligned}
L_{e} V(x) \delta(x) & \leq\left\|L_{e}^{\prime} V(x)\right\|\|\delta(x)\| \\
& \leq\left\|L_{e}^{\prime} V(x)\right\|\|n(x)\|, \quad \forall \delta(x) \in \Gamma,
\end{aligned}
$$


which reduces to

$$
\begin{aligned}
L_{f+\Delta f} V(x)+\frac{1}{2} l^{\prime} \frac{\partial^{2} V(x)}{\partial x^{2}} l= & L_{f} V(x)+L_{e} V(x) \delta(x) \\
& +\frac{1}{2} l^{\prime} \frac{\partial^{2} V(x)}{\partial x^{2}} l \leq 0 .
\end{aligned}
$$

The robust KYP property of system (1) is guaranteed through Definition 2. Then, by Itô's formula, we have

$$
\begin{aligned}
\mathscr{L} V(x)= & L_{f} V(x)+L_{\Delta f} V(x)+L_{g} V(x) u \\
& +\frac{1}{2} l^{\prime} \frac{\partial^{2} V(x)}{\partial x^{2}} l \leq L_{g} V(x) u,
\end{aligned}
$$

where $\mathscr{L}$ is the infinitesimal generator of system (1). From the second equation in (6), it follows that

$$
\mathscr{L} V(x) \leq y^{\prime} u .
$$

Integrating the above inequality from $s$ to $t$ for any $t \geq s \geq 0$, $x(s) \in R^{n}$, and for all admissible $\Delta f(x)$,

$$
E V(x(t))-V(x(s)) \leq E \int_{s}^{t} y^{\prime}(\tau) u(\tau) d \tau,
$$

which implies that system (1) is robust passive by Definition 1 .

Necessity. Assume that system (1) is robust passive which sustains a storage function $V(x)$; that is, (11) always holds for $\forall \delta(x) \in \Gamma$. By (11) with any $x(s) \in R^{n}$, we have

$$
\frac{E V(x(t))-V(x(s))}{t-s}-\frac{E \int_{s}^{t} y^{\prime}(\tau) u(\tau) d \tau}{t-s} \leq 0, \quad \forall t>s .
$$

By Itô’s formula,

$$
\begin{aligned}
& E V(x(t))-V(x(s)) \\
& =E \int_{s}^{t}\left(L_{f} V(x)+L_{e} V(x) \delta(x)\right. \\
& \left.\quad+L_{g} V(x) u+\frac{1}{2} l^{\prime} \frac{\partial^{2} V(x)}{\partial x^{2}} l\right) d \tau .
\end{aligned}
$$

Let $t \downarrow s$, it follows that

$$
\begin{gathered}
L_{f} V(x)+L_{e} V(x) \delta(x)+\frac{1}{2} l^{\prime} \frac{\partial^{2} V(x)}{\partial x^{2}} l \\
+\left(L_{g}^{\prime} V(x)-y\right)^{\prime} u \leq 0
\end{gathered}
$$

always holds for any control input $u$, which implies that (5) holds; that is, the robust passive system (1) has the robust KYP property.

Denote

$$
\alpha(x)=L_{f} V(x)+\frac{1}{2} l^{\prime} \frac{\partial^{2} V(x)}{\partial x^{2}} l, \quad \beta^{\prime}(x)=L_{e} V(x) .
$$

So,

$$
\begin{aligned}
& L_{f+\Delta f} V(x)+\frac{1}{2} l^{\prime} \frac{\partial^{2} V(x)}{\partial x^{2}} l \\
& \quad=\alpha(x)+\beta^{\prime}(x) \delta(x) \leq 0, \quad \forall \delta(x) \in \Gamma .
\end{aligned}
$$

The rest is similar to the proof of Lemma 2 in [8], and we only note that through defining

$$
\delta_{0}=\frac{\|n(x)\|}{\|\beta(x)\|} \beta(x), \quad \beta(x) \neq 0,
$$

it can be found that $\delta_{0} \in \Gamma$, which also deduces that

$$
\left|\beta^{\prime}(x) \delta_{0}(x)\right|=\beta^{\prime}(x) \beta(x) \frac{\|n(x)\|}{\|\beta(x)\|}=\|\beta(x)\|\|n(x)\| .
$$

Obviously, the above equality also holds when $\beta(x)=0$. Considering the fact that

$$
\sup _{\delta \in \Gamma}\left|\beta^{\prime}(x) \delta(x)\right| \leq\|\beta(x)\|\|n(x)\|,
$$

we get to the result that

$$
\sup _{\delta \in \Gamma}\left|\beta^{\prime}(x) \delta(x)\right|=\|\beta(x)\|\|n(x)\| .
$$

Hence, from (16) and (20), we have

$$
\alpha(x) \leq-\sup _{\delta \in \Gamma}\left|\beta^{\prime}(x) \delta(x)\right|=-\|\beta(x)\|\|n(x)\|,
$$

which also implies that

$$
\alpha(x)+\|\beta(x)\|\|n(x)\|
$$

$$
=L_{f} V(x)+\left\|L_{e}^{\prime} V(x)\right\|\|n\|+\frac{1}{2} l^{\prime} \frac{\partial^{2} V(x)}{\partial x^{2}} l \leq 0 .
$$

The proof of Theorem 3 is completed.

Remark 4. Indeed, Theorem 3 has established the equivalent relations between (4), (5), and (6). Obviously, the condition (6) is more convenient than (4) or (5), which has taken the bounding function $n(x)$ instead of the unknown function $\delta(x)$.

Remark 5. From the proof of Theorem 3, we can also discuss the robust strict passivity for system (1). There is no difference except that the inequality in (6) becomes a strict inequality which holds for a positive definite function $V(x)$. Moreover, it is easy to deduce from Theorem 3 that a sufficient condition for the robust passivity of system (1) is that there exists a realvalued function $\lambda(x)>0$, such that

$$
\begin{gathered}
L_{f} V(x)+\frac{\lambda}{2}\left\|L_{e} V(x)\right\|^{2}+\frac{1}{2 \lambda}\|n(x)\|^{2}+\frac{1}{2} l^{\prime} \frac{\partial^{2} V(x)}{\partial x^{2}} l \leq 0, \\
L_{g} V(x)=h^{\prime} .
\end{gathered}
$$


Indeed, if (23) is also a strict inequality, it is equivalent to the strict inequalities (5) or (6). The proof can follow the line of Theorem 3 and [8] and is omitted.

In what follows, we recall some facts in the theory of stochastic stability, where only global stability is considered. Obviously, local stability results may also be achieved in a similar way.

Consider the uncertain stochastic unforced system with

$$
\begin{array}{r}
d x=(f(x)+\Delta f(x)) d t+l(x) d w, \\
x(0)=x_{0} \in R^{n}, \quad f(0)=0, \\
\Delta f(0)=0, \quad l(0)=0 .
\end{array}
$$

Definition 6 (see [20]). System (24) with the structural uncertainty is said to be stable in probability if for any $\varepsilon>0$,

$$
\lim _{x \rightarrow 0} P\left(\sup _{t \geq 0}\|x(t)\|>\varepsilon\right)=0 .
$$

Additionally, if we also have $P\left(\lim _{t \rightarrow \infty} x(t)=0\right)=1$ for $\forall x_{0} \in R^{n}$, system (24) is said to be GASP.

Definition 7. System (24) with the structural uncertainty is called zero-state detectable if for all $x(0)=x_{0} \in R^{n}$,

$$
\begin{aligned}
& h(x(t))=0, \\
& \text { a.s. } \forall t \geq 0 \Longrightarrow P\left(\lim _{t \rightarrow \infty} x(t)=0, x(0)=x_{0}\right)=1 .
\end{aligned}
$$

System (24) is globally zero-state observable if for all $x_{0} \in R^{n}$, $h(x(t)) \equiv 0$ implies $x_{0} \equiv 0$.

\section{Feedback Equivalence and Global Stabilization}

In this section, we discuss the feedback equivalence and global stabilization problems for the general nonlinear stochastic system (1) based on the above robust passivity theory. Similarly as the deterministic system case, we first present the following relative degree definition for uncertain nonlinear stochastic systems.

Definition 8 (relative degree). The nonlinear stochastic system (1) is said to have the relative degree $\rho$ if

$$
\begin{aligned}
& L_{g} L_{f}^{j-1} h(x)=0, \\
& L_{g} L_{f}^{\rho-1} h(x) \neq 0,
\end{aligned}
$$

where $j=1,2, \ldots, \rho-1$.

In what follows, we only consider the simple case of $\rho=1$. For system (1), by Itô's formula, we know that

$$
\begin{aligned}
d y & =\frac{\partial h^{\prime}}{\partial x}(d x)+\frac{1}{2}(d x)^{\prime} \frac{\partial^{2} h}{\partial x^{2}}(d x) \\
& =\left(L_{f} h+L_{\Delta f} h+L_{g} h u+\frac{1}{2} l^{\prime} \frac{\partial^{2} h}{\partial x^{2}} l\right) d t+L_{l} h d w .
\end{aligned}
$$

From the above assumption, we have $L_{g} h(x) \neq 0$. If we take $u$ as

$$
u=\left(L_{g} h\right)^{-1}\left(v-L_{f} h-\frac{1}{2} l^{\prime} \frac{\partial^{2} h}{\partial x^{2}} l\right),
$$

where $v$ can be regarded as a new control input instead of $u$. Then, we obtain that

$$
d y=v d t+L_{\Delta f} h d t+L_{l} h d w
$$

Motivated by the works of $[5,8]$, we assume that the vector fields $\widetilde{g}_{1}(x), \widetilde{g}_{2}(x), \ldots, \widetilde{g}_{p}(x)$ are complete, where $\left[\widetilde{g}_{1}(x), \ldots, \widetilde{g}_{p}(x)\right]=g(x)\left[L_{g} h(x)\right]^{-1}$ and the distribution spanned by $\tilde{g}_{1}(x), \tilde{g}_{2}(x), \ldots, \tilde{g}_{p}(x)$ is involutive. Under these hypotheses, it is possible to find a global diffeomorphism $\phi(x)$, such that

$$
\xi=\phi(x)=\left[\begin{array}{l}
\theta(x) \\
h(x)
\end{array}\right]=\left[\begin{array}{l}
\eta \\
y
\end{array}\right]
$$

where $\eta=\theta(x) \in R^{n-1}$. Then, system (1) can be transformed into an interconnected system of the form

$$
\begin{gathered}
d \eta=\left(L_{f} \theta+L_{\Delta f} \theta+\frac{1}{2} l^{\prime} \frac{\partial^{2} \theta}{\partial x^{2}} l\right) d t+L_{l} \theta d w \\
d y=v d t+L_{\Delta f} h d t+L_{l} h d w .
\end{gathered}
$$

For simplicity, we adopt that $\tilde{h}(\xi)=h\left(\phi^{-1}(\xi)\right)$. Then, by taking the transformation $x=\phi^{-1}(\xi)$, system (1), in the new coordinate, is formulated as

$$
\begin{gathered}
d \eta=\tilde{f}(\xi) d t+\widetilde{e}(\xi) \widetilde{\delta}(\xi) d t+\widetilde{l}(\xi) d w, \\
d y=v d t+\widehat{e}(\xi) \widetilde{\delta} d t+\widehat{l}(\xi) d w,
\end{gathered}
$$

where

$$
\begin{gathered}
\widetilde{f}(\xi)=L_{f} \theta+\left.\frac{1}{2} l^{\prime} \frac{\partial^{2} \theta}{\partial x^{2}} l\right|_{x=\phi^{-1}(\xi)}, \quad \tilde{e}(\xi)=\left.L_{e} \theta\right|_{x=\phi^{-1}(\xi)}, \\
\tilde{\delta}(\xi)=\delta\left(\phi^{-1}(\xi)\right), \quad \tilde{l}(\xi)=\left.L_{l} \theta\right|_{x=\phi^{-1}(\xi)}, \\
\widehat{e}_{i}(\xi)=\left.L_{e} h\right|_{x=\phi^{-1}(\xi)}, \quad \widehat{l}_{i}(\xi)=\left.L_{l} h\right|_{x=\phi^{-1}(\xi)} .
\end{gathered}
$$


Here, we define

$$
\begin{aligned}
& \tilde{f}_{1}(\xi)=\left.\int_{0}^{1} \frac{\partial \tilde{f}(\eta, \zeta)}{\partial \zeta}\right|_{\zeta=\tau y} d \tau, \\
& \tilde{e}_{1}(\xi)=\left.\int_{0}^{1} \frac{\partial \widetilde{e}(\eta, \zeta)}{\partial \zeta}\right|_{\zeta=\tau y} d \tau, \\
& \tilde{l}_{1}(\xi)=\left.\int_{0}^{1} \frac{\partial \tilde{l}(\eta, \zeta)}{\partial \zeta}\right|_{\zeta=\tau y} d \tau, \\
& \widehat{e}_{1}(\xi)=\left.\int_{0}^{1} \frac{\partial \widehat{e}(\eta, \zeta)}{\partial \zeta}\right|_{\zeta=\tau y} d \tau, \\
& \widehat{l}_{1}(\xi)=\left.\int_{0}^{1} \frac{\partial \widehat{l}(\eta, \zeta)}{\partial \zeta}\right|_{\zeta=\tau y} d \tau .
\end{aligned}
$$

Then, the following decomposition holds

$$
\tilde{f}(\xi)=\tilde{f}_{*}(\eta)+\tilde{f}_{1}(\xi) y \text {, where } \tilde{f}_{*}(\eta)=\tilde{f}(\eta, 0) \text {. }
$$

Similarly, we can also find $\widetilde{l}, \widehat{l}, \widetilde{e}$, and $\widetilde{n}$ with the following structures, respectively

$$
\begin{array}{ll}
\tilde{l}(\xi)=\tilde{l}_{*}(\eta)+\tilde{l}_{1}(\xi) y, & \tilde{e}(\xi)=\tilde{e}_{*}(\eta)+\tilde{e}_{1}(\xi) y, \\
\widehat{l}(\xi)=\widehat{l}_{*}(\eta)+\widehat{l}_{1}(\xi) y, & \tilde{n}(\xi)=n(\eta, 0)+\tilde{n}_{1}(\xi) y .
\end{array}
$$

Moreover, we set the control input as

$$
v=c(\xi)+\omega
$$

where $c$ is a smooth function defined on $R^{n}$ with $c(0)=0$, and $\omega$ is the new control input. Equivalently, system (33) can be expressed with the form of

$$
\begin{gathered}
d \xi=F(\xi) d t+E(\xi) \widetilde{\delta} d t+G(\xi) \omega d t+L(\xi) d w, \\
y=\widetilde{h}(\xi)
\end{gathered}
$$

where

$$
\begin{array}{cc}
F(\xi)=\left[\begin{array}{c}
\widetilde{f}(\xi) \\
c(\xi)
\end{array}\right], & E(\xi)=\left[\begin{array}{l}
\widetilde{e}(\xi) \\
\widehat{e}(\xi)
\end{array}\right], \\
G(\xi)=\left[\begin{array}{l}
0 \\
I
\end{array}\right], & L(\xi)=\left[\begin{array}{l}
\widetilde{l}(\xi) \\
\widehat{l}(\xi)
\end{array}\right] .
\end{array}
$$

Let zero-output system describe the internal dynamic of a system which is consistent with the constraint $y \equiv$ 0 . Obviously, the zero-output dynamic of system (1) comes down to

$$
d \eta=\widetilde{f}_{*}(\eta) d t+\widetilde{e}_{*}(\eta) \widetilde{\delta}(\eta, 0)+\widetilde{l}_{*}(\eta) d w .
$$

As follows, we define the minimum phase system for nonlinear stochastic system (1).
Definition 9. System (1) is said to be

(i) Robust weakly minimum phase if the zero-output system (41) is stable in probability; that is, there exists a nonnegative function $W(\eta) \in \mathscr{C}^{2}: R^{n-1} \rightarrow R^{+}$with $W(0)=0$, satisfying the following Lyapunov inequality:

$$
L_{\tilde{f}_{*}} W(\eta)+\left\|L_{\widetilde{e}_{*}}^{\prime} W(\eta)\right\|\|n(\eta, 0)\|+\frac{1}{2} \widetilde{l}_{*}^{\prime} \frac{\partial^{2} W(\eta)}{\partial \eta^{2}} \widetilde{l}_{*} \leq 0 .
$$

(ii) Robust minimum phase if for the zero-output system (41) is GASP; that is, there exists a positive definite function $W(\eta) \in \mathscr{C}^{2}: R^{n-1} \rightarrow R^{+}$with $W(0)=0$, satisfying the following Lyapunov inequality:

$$
L_{\tilde{f}_{*}} W(\eta)+\left\|L_{\widetilde{e}_{*}}^{\prime} W(\eta)\right\|\|n(\eta, 0)\|+\frac{1}{2} \tilde{l}_{*} \frac{\partial^{2} W(\eta)}{\partial \eta^{2}} \widetilde{l}_{*}<0 .
$$

(iii) Robust strongly minimum phase, if for the zerooutput system (41) there exists positive definite functions $W(\eta) \in \mathscr{C}^{2}: R^{n-1} \rightarrow R^{+}$with $W(0)=0$, and $\lambda(\xi):$ $R^{n} \rightarrow R^{+}$, satisfying the following Lyapunov-like inequality constraint:

$$
\begin{gathered}
L_{\tilde{f}_{*}} W(\eta)+\frac{\lambda}{2}\left\|L_{\tilde{e}_{*}} W(\eta)\right\|^{2}+\frac{1}{2 \lambda}\|n(\eta, 0)\|^{2} \\
+\frac{1}{2} \widetilde{l}_{*} \frac{\partial^{2} W(\eta)}{\partial \eta^{2}} \widetilde{l}_{*} \leq-\frac{1}{2} \widehat{l}_{*}^{\prime} \widehat{l}_{*} .
\end{gathered}
$$

The following theorem discusses the relationship between the passivity of system (39) and the stability of the zero-output system (41).

Theorem 10. If system (1) is robust strongly minimum phase, then the system is feedback equivalent to a robust passive system (39). Conversely, the robust passivity of system (39) with a positive storage function implies that system (1) is robust weakly minimum phase.

Proof. We construct the storage function $V(\xi)=W(\eta)+$ $(1 / 2) y^{\prime} y$ for system (39), where $W(\eta)$ satisfies the inequality (44). Obviously, we have

$$
\begin{aligned}
L_{F} V(\xi) & +\left\|L_{E}^{\prime} V(\xi)\right\|\|n(\xi)\|+\frac{1}{2} L^{\prime} \frac{\partial^{2} V(\xi)}{\partial \xi^{2}} L \\
\leq & L_{F} V(\xi)+\frac{\lambda}{2}\left\|L_{E} V(\xi)\right\|^{2}+\frac{1}{2 \lambda}\|n(\xi)\|^{2} \\
& +\frac{1}{2} L^{\prime} \frac{\partial^{2} V(\xi)}{\partial \xi^{2}} L
\end{aligned}
$$




$$
\begin{aligned}
& =L_{\tilde{f}} W(\eta)+y^{\prime} c+\frac{\lambda}{2}\left\|L_{\widetilde{e}} W(\eta)+y^{\prime} \hat{e}\right\|^{2} \\
& +\frac{1}{2 \lambda}\|n(\xi)\|^{2}+\frac{1}{2} \tilde{l}^{\prime} \frac{\partial^{2} W(\eta)}{\partial \eta^{2}} \tilde{l}+\frac{1}{2} \hat{l} \hat{l} \\
& =L_{\tilde{f}_{*}+\tilde{f}_{1} y} W(\eta)+y^{\prime} c \\
& +\frac{\lambda}{2}\left\|L_{\widetilde{e}_{*}} W(\eta)+\widetilde{e}_{1} y+y^{\prime} \hat{e}\right\|^{2} \\
& +\frac{1}{2 \lambda}\left\|n(\eta, 0)+n_{1}(\xi) y\right\|^{2} \\
& +\frac{1}{2}\left(\tilde{l}_{*}+\widetilde{l}_{1} y\right)^{\prime} \frac{\partial^{2} W(\eta)}{\partial \eta^{2}}\left(\widetilde{l}_{*}+\tilde{l}_{1} y\right) \\
& +\frac{1}{2}\left(\widehat{l}_{*}+\widehat{l}_{1} y\right)^{\prime}\left(\widehat{l}_{*}+\widehat{l}_{1} y\right) \\
& =L_{\tilde{f}_{*}} W(\eta)+L_{\tilde{f}_{1}} W(\eta) y+y^{\prime} c \\
& +\left\|L_{\tilde{e}_{*}} W(\eta)\right\|^{2}+\frac{\lambda}{2} y^{\prime} c_{1}+\frac{1}{2 \lambda}\|n(\eta, 0)\|^{2} \\
& +\frac{1}{2 \lambda} y^{\prime} c_{2}+\frac{1}{2} \widetilde{l}_{*} \frac{\partial^{2} W(\eta)}{\partial \eta^{2}} \widetilde{l}_{*} \\
& +y^{\prime}\left(\tilde{l}_{1} \frac{\partial^{2} W(\eta)}{\partial \eta^{2}} \widetilde{l}_{*}+\frac{1}{2} \widetilde{l}_{1}^{\prime} \frac{\partial^{2} W(\eta)}{\partial \eta^{2}} \widetilde{l}_{1} y\right) \\
& +\frac{1}{2} \hat{l}_{*} \hat{l}_{*}+y^{\prime}\left(\hat{l}_{1} \hat{l}_{*}+\frac{1}{2} \hat{l}_{1} \hat{l}_{1} y\right) \\
& =L_{\widetilde{f}_{*}} W(\eta)+\left\|L_{\widetilde{e}_{*}} W(\eta)\right\|^{2}+\frac{1}{2 \lambda}\|n(\eta, 0)\|^{2} \\
& +\frac{1}{2} \widetilde{l}_{*} \frac{\partial^{2} W(\eta)}{\partial \eta^{2}} \tilde{l}_{*}+\frac{1}{2} \hat{l}_{*} \widehat{l}_{*} \\
& +y^{\prime}\left(L_{\widetilde{f}_{1}}^{\prime} W(\eta)+c+\frac{\lambda}{2} c_{1}+\frac{1}{2 \lambda} c_{2}\right. \\
& +\widetilde{l}_{1} \frac{\partial^{2} W(\eta)}{\partial \eta^{2}} \tilde{l}_{*}+\frac{1}{2} \tilde{l}_{1} \frac{\partial^{2} W(\eta)}{\partial \eta^{2}} \tilde{l}_{1} y \\
& \left.+\hat{l}_{1} \hat{l}_{*}+\frac{1}{2} \hat{l}_{1} \hat{l}_{1}\right),
\end{aligned}
$$

where

$$
\begin{gathered}
c_{1}=2\left(L_{\widetilde{e}_{1}} W+\widehat{e}\right)^{\prime} L_{\tilde{e}_{*}}^{\prime} W+\left(L_{\widetilde{e}_{1}} W+\widehat{e}\right)\left(L_{\widetilde{e}_{1}} W+\widehat{e}\right)^{\prime} y, \\
c_{2}=\left(n_{1}\right)^{\prime}\left(2 n(\eta, 0)+n_{1} y\right) .
\end{gathered}
$$

For (45), taking

$$
\begin{aligned}
c= & -L_{\tilde{f}_{1}}^{\prime} W-\frac{\lambda}{2} c_{1}-\frac{1}{2 \lambda} c_{2}-\widetilde{l}_{1}^{\prime} \frac{\partial^{2} W}{\partial \eta^{2}} \widetilde{l}_{*} \\
& -\frac{1}{2} \widetilde{l}_{1} \frac{\partial^{2} W}{\partial \eta^{2}} \widetilde{l}_{1} y-\hat{l}_{1} \widehat{l}_{*}-\frac{1}{2} \hat{l}_{1} \widehat{l}_{1},
\end{aligned}
$$

and considering the robust strongly minimum phase property, we have

$$
L_{F} V(\xi)+\left\|L_{E}^{\prime} V(\xi)\right\|\|n(\xi)\|+\frac{1}{2} L^{\prime} \frac{\partial^{2} V(\xi)}{\partial \xi^{2}} L \leq 0 .
$$

Besides, it is obvious that

$$
L_{G} V(\xi)=\left[\frac{\partial W^{\prime}}{\partial \eta}, y^{\prime}\right]\left[\begin{array}{l}
0 \\
I
\end{array}\right]=\widetilde{h}^{\prime}
$$

Then, by Theorem 3, we know that system (39) is robust passive.

On the other hand, suppose that there is a feedback control law $v_{*}$ that renders the closed-loop system (39) robust passive with a storage function $V(\xi)$, which is positive definite and satisfies

$$
\begin{gathered}
L_{F} V(\xi)+\left\|L_{E}^{\prime} V(\xi)\right\|\|n(\xi)\|+\frac{1}{2} L^{\prime} \frac{\partial^{2} V(\xi)}{\partial \xi^{2}} L \leq 0, \\
L_{G} V(\xi)=\tilde{h}^{\prime} .
\end{gathered}
$$

Note that

$$
\begin{gathered}
\frac{\partial V^{\prime}(\xi)}{\partial \xi}=\left[\frac{\partial V^{\prime}(\xi)}{\partial \eta}, \frac{\partial V^{\prime}(\xi)}{\partial y}\right], \\
\frac{\partial^{2} V(\xi)}{\partial \xi^{2}}=\left[\begin{array}{ll}
\frac{\partial^{2} V(\xi)}{\partial \eta^{2}} & \frac{\partial^{2} V(\xi)}{\partial \eta y} \\
\frac{\partial^{2} V(\xi)}{\partial y \eta} & \frac{\partial^{2} V(\xi)}{\partial y^{2}}
\end{array}\right] .
\end{gathered}
$$

Then, (50) deduces that

$$
\begin{aligned}
& \frac{\partial V^{\prime}(\xi)}{\partial \eta} \widetilde{f}(\xi)+\frac{\partial V^{\prime}(\xi)}{\partial y} c(\xi) \\
& +\left\|\left(\frac{\partial V^{\prime}(\xi)}{\partial \eta} \widetilde{e}(\xi)+\frac{\partial V^{\prime}(\xi)}{\partial y} \widehat{e}(\xi)\right)^{\prime}\right\|\|n(\xi)\| \\
& +\frac{1}{2}\left[\begin{array}{l}
\widetilde{l} \\
\widehat{l}
\end{array}\right]^{\prime}\left[\begin{array}{ll}
\frac{\partial^{2} V(\xi)}{\partial \eta^{2}} & \frac{\partial^{2} V(\xi)}{\partial \eta y} \\
\frac{\partial^{2} V(\xi)}{\partial y \eta} & \frac{\partial^{2} V(\xi)}{\partial y^{2}}
\end{array}\right]\left[\begin{array}{l}
\tilde{l} \\
\widehat{l}
\end{array}\right] \leq 0
\end{aligned}
$$

holds for all $\xi \in R^{n}$. Obviously,

$$
\frac{\partial V(\eta, 0)}{\partial y}=0, \quad \frac{\partial^{2} V(\eta, 0)}{\partial y^{2}}=0, \quad \forall \eta \in R^{n-1} .
$$

Setting $y=0$ in (52), it reduces to

$$
\begin{gathered}
L_{\tilde{f}_{*}} V(\eta, 0)+\left\|L_{\tilde{e}_{*}}^{\prime} V(\eta, 0)\right\|\|n(\eta, 0)\| \\
+\frac{1}{2} \widetilde{l}_{*} \frac{\partial^{2} V(\eta, 0)}{\partial \eta^{2}} \tilde{l}_{*} \leq 0 .
\end{gathered}
$$

Then, by [20] and Definition 9(i), we know that the zerooutput system (41) is stable in probability, and that system (1) is robust weakly minimum phase. The proof of Theorem 10 is completed. 
Corollary 11. If the zero-output system (41) satisfies the following Lyapunov-like equality constraint, that is, there exists a positive definite and proper function $W(\eta) \in \mathscr{C}^{2}: R^{n-1} \rightarrow$ $R^{+}$with $W(0)=0$ and $\lambda(\xi): R^{n} \rightarrow R^{+}$, such that

$$
\begin{aligned}
& L_{\widetilde{f}_{*}} W(\eta)+\frac{\lambda}{2}\left\|L_{\widetilde{e}_{*}} W(\eta)\right\|^{2}+\frac{1}{2 \lambda}\|n(\eta, 0)\|^{2} \\
& +\frac{1}{2} \vec{l}_{*} \frac{\partial^{2} W(\eta)}{\partial \eta^{2}} \widetilde{l}_{*}<-\frac{1}{2} \hat{l}_{*} \widehat{l}_{*},
\end{aligned}
$$

then system (1) is feedback equivalent to a robust strictly passive system (39). Conversely, the robust strict passivity of system (39) with a positive definite and proper storage function implies system (1) to be robust minimum phase.

Proof. From Remark 5 and the proof of Theorem 10, the above conclusion is obvious.

As follows, we use the above results to study the problem of global stabilization for system (39).

Theorem 12. Suppose that system (1) is robust strongly minimum phase which sustains a positive definite and proper function $W(\eta) \in \mathscr{C}^{2}: R^{n-1} \rightarrow R^{+}$with $W(0)=0$ and $a$ positive definition function $\lambda(\xi): R^{n} \rightarrow R^{+}$satisfying (44), and that system (39) is zero-state detectable, then system (39) is globally asymptotically stabilizable in probability.

Proof. We construct the storage function $V(\eta, y)=W(\eta)+$ $(1 / 2) y^{\prime} y$ for system (39), where $W(\eta)$ satisfies (44). Obviously, we have

$$
\begin{aligned}
\mathscr{L}_{\omega} V= & L_{F} V(\xi)+L_{E} V(\xi) \widetilde{\delta}+L_{G} V(\xi) \omega+\frac{1}{2} L^{\prime} \frac{\partial^{2} V(\xi)}{\partial \xi^{2}} L \\
\leq & L_{F} V(\xi)+L_{G} V(\xi) \omega+\left\|L_{E}^{\prime} V(\xi)\right\|\|n(\xi)\| \\
& +\frac{1}{2} L^{\prime} \frac{\partial^{2} V(\xi)}{\partial \xi^{2}} L \\
\leq & L_{F} V(\xi)+L_{G} V(\xi) \omega+\frac{1}{2 \lambda}\left\|L_{E} V(\xi)\right\|^{2} \\
& +\frac{1}{2 \lambda}\|n(\xi)\|^{2}+\frac{1}{2} L^{\prime} \frac{\partial^{2} V(\xi)}{\partial \xi^{2}} L,
\end{aligned}
$$

where $\mathscr{L}_{\omega}$ is the infinitesimal generator of system (39). Similar as the proof of Theorem 10, we have

$$
\begin{aligned}
\mathscr{L}_{\omega} V \leq & L_{\tilde{f}_{*}} W(\eta)+\left\|L_{\widetilde{e}_{*}} W(\eta)\right\|^{2}+\frac{1}{2 \lambda}\|n(\eta, 0)\|^{2} \\
& +\frac{1}{2} \widetilde{l}_{*} \frac{\partial^{2} W(\eta)_{\tilde{l}_{*}}+\frac{1}{2} \hat{l}_{*} \widehat{l}_{*}}{\partial \eta^{2}} \\
& +y^{\prime}\left(\omega+c+\frac{\lambda}{2} c_{1}+\frac{1}{2 \lambda} c_{2}+L_{\widetilde{f}_{1}}^{\prime} W(\eta)\right.
\end{aligned}
$$

$$
\begin{aligned}
& +\vec{l}_{1} \frac{\partial^{2} W(\eta)}{\partial \eta^{2}} \tilde{l}_{*}+\frac{1}{2} \tilde{l}_{1} \frac{\partial^{2} W(\eta)}{\partial \eta^{2}} \tilde{l}_{1} y \\
& \left.+\hat{l}_{1} \hat{l}_{*}+\frac{1}{2} \hat{l}_{1}^{\prime} \hat{l}_{1}\right) .
\end{aligned}
$$

Taking the same $c_{1}, c_{2}$ and the corresponding $c$ in (46)-(47) as in Theorem 10, we have

$$
\mathscr{L}_{\omega} V \leq y^{\prime} \omega .
$$

Let $\psi(y): R \rightarrow R$ be any smooth function satisfying $\psi(0)=$ 0 and $y^{\prime} \psi(y)>0$ for each nonzero $y$. Then, we can construct the control law as $\omega=-\psi(y)$, which follows that

$$
\mathscr{L}_{\omega=-\psi(y)} V \leq-y^{\prime} \psi(y) \leq 0 .
$$

The rest is proved by using the version of LaSalle's invariance principle [21], which is similar to Theorem 4.6 of [12] and is omitted. The proof of Theorem 12 is completed.

Corollary 13. If the zero-output system (41) satisfies the Lyapunov-like equality constraint (55) with a positive definite and proper function $W(\eta) \in \mathscr{C}^{2}: R^{n-1} \rightarrow R^{+}$with $W(0)=$ 0 , then system (39) is globally asymptotically stabilizable in probability.

Proof. The proof is similar to that of Theorem 12, and we only note that

$$
\mathscr{L}_{\omega=-\psi(y)} V \leq-y^{\prime} \psi(y)-S<0 .
$$

Remark 14. Obviously, if the conditions of Theorem 12 or Corollary 13 are satisfied, then system (39) is GASP under the control law $\omega=-y$. Furthermore, system (1) is GASP under the corresponding control

$$
u=\left(L_{g} h\right)^{-1}\left(-y+\left.c(\xi)\right|_{\xi=\phi(x)}-L_{f} h-\frac{1}{2} l^{\prime} \frac{\partial^{2} h}{\partial x^{2}} l\right) .
$$

Remark 15. In this work, we only consider the relative degree $\rho=1$ case. Further efforts should be concentrated on the robust passive control design for uncertain nonlinear stochastic system with any arbitrary relative degree case. In that case, we need to discuss the robust passivity through applying the backstepping technique for nonlinear stochastic systems.

\section{Numerical Example}

Consider the following nonlinear stochastic system:

$$
\begin{gathered}
d x=\left[\begin{array}{l}
x_{1} x_{2}-2 x_{1} \\
8 x_{1}^{3}+x_{1} x_{2}
\end{array}\right] d t+\left[\begin{array}{l}
x_{1} \\
x_{2}
\end{array}\right] \delta(x) d t \\
+\left[\begin{array}{l}
1 \\
1
\end{array}\right] u d t+\left[\begin{array}{l}
x_{1} \\
x_{2}
\end{array}\right] d w, \\
y=x_{1}+x_{2} .
\end{gathered}
$$


The uncertainty $\delta(x)$ satisfies

$$
\|\delta(x)\| \leq\|n(x)\|
$$

where

$$
n(x)=k x_{1}^{2}
$$

and $k$ is a given constant satisfying $0<k \leq 1$.

Firstly, we construct the function $\phi(x)$ as

$$
\xi=\phi(x)=\left[\begin{array}{l}
\theta(x) \\
h(x)
\end{array}\right]=\left[\begin{array}{l}
x_{1}-x_{2} \\
x_{1}+x_{2}
\end{array}\right]=\left[\begin{array}{l}
\eta \\
y
\end{array}\right],
$$

then it is obvious that the following holds

$$
\phi^{-1}(\eta, y)=\left[\begin{array}{l}
x_{1} \\
x_{2}
\end{array}\right]=\left[\begin{array}{c}
\frac{1}{2}(\eta+y) \\
\frac{1}{2}(-\eta+y)
\end{array}\right] \text {. }
$$

Also, it can be found that $\left(\partial h^{\prime} / \partial x\right) g=2,\left(\partial \theta^{\prime} / \partial x\right) g=0$ hold for the above system. Thus, all the assumptions are satisfied. According to the discussion in Section 3, we design the controller $u$ as

$$
u=v-8 x_{1}^{3}-2 x_{1} x_{2}+2 x_{1} \text {. }
$$

Then, we change system (62) into the following form

$$
\begin{gathered}
d \eta=\left(-(\eta+y)^{3}-\eta-y+\eta \widetilde{\delta}\right) d t+\eta d w \\
d y=(v+y \widetilde{\delta}) d t+y d w
\end{gathered}
$$

with

$$
\begin{aligned}
\tilde{f}_{*}=-\eta^{3}-\eta, & \tilde{f}_{1}=-1, \\
\tilde{e}_{*}=\eta, & \tilde{e}_{1}=0, \\
\widetilde{l}_{*}=\eta, & \widetilde{l}_{1}=0, \\
\widehat{e}_{*}=0, & \widehat{e}_{1}=\frac{1}{2}, \\
\widehat{l}_{*}=0, & \widehat{l}_{1}=\frac{1}{2}, \\
\tilde{n}(\eta, 0)=\frac{k}{4} \eta^{2}, & \tilde{n}_{1}=\frac{k}{4}(2 \eta+y) .
\end{aligned}
$$

Taking $W=\eta^{2}$, then from (44), we obtain that

$$
\begin{aligned}
L_{\tilde{f}_{*}} W(\eta) & +\frac{\lambda}{2}\left\|L_{\widetilde{e}_{*}} W(\eta)\right\|^{2} \\
& +\frac{1}{2 \lambda}\|n(\eta, 0)\|^{2}+\frac{1}{2} \widetilde{l}_{*} \frac{\partial^{2} W(\eta)}{\partial \eta^{2}} \widetilde{l}_{*}+\frac{1}{2} \widehat{l}_{*}^{\prime} \widehat{l}_{*} \\
= & 2 \eta\left(-\eta^{3}-\eta\right)+2 \lambda \eta^{4}+\frac{k^{2}}{32 \lambda} \eta^{4}+\eta^{2} .
\end{aligned}
$$

Leting $k=1$ and $\lambda=1 / 2$, we have

$$
-\frac{15}{16} \eta^{4}-\eta^{2} \leq 0
$$

Hence, system (62) is robust strongly minimum phase. By Theorem 10, we know that system (62) is feedback equivalent to the robust passive system (68), and we have

$$
\begin{gathered}
c_{1}=2 \eta^{2} y+\frac{1}{4} y^{3}, \\
c_{2}=\frac{1}{4} \eta^{3}+\frac{3}{8} \eta^{2} y+\frac{1}{4} \eta y^{2}+\frac{1}{16} y^{3} .
\end{gathered}
$$

Then, from (47), we have

$$
c=-\frac{1}{4} \eta^{3}-\frac{1}{8} y^{3}-\frac{7}{8} \eta^{2} y-\frac{1}{4} \eta y^{2}+2 \eta-\frac{1}{8} .
$$

Besides, according to Theorem 12 and Remark 14, we can construct the control law as $\omega=-y$, and it follows that

$$
\begin{aligned}
v= & c(\eta, y)-y=-\frac{1}{4} \eta^{3}-\frac{1}{8} y^{3} \\
& -\frac{7}{8} \eta^{2} y-\frac{1}{4} \eta y^{2}+2 \eta-y-\frac{1}{8},
\end{aligned}
$$

which guarantees that the closed-loop system (68) is GASP.

\section{Conclusion}

In this paper, we have discussed the robust passivity, feedback equivalence, and global stabilization problems for a class of uncertain nonlinear stochastic systems, which contain the structural uncertainty. Through establishing the robust passivity theory, a robust stochastic version of KYP lemma has been presented for such a class of systems. Then, the feedback equivalence and global stabilization problems have been discussed through the robust strongly minimum phase property. However, more efforts should be concentrated on the robust passive control of uncertain nonlinear stochastic systems with any arbitrary relative degree.

\section{Acknowledgments}

This work is partially supported by the National Basic Research Program of China (973 Program) (Grant no. 2012CB215203), the National Natural Science Foundation of China (no. 61203043 and no. 51036002), and the Fundamental Research Funds for the Central Universities.

\section{References}

[1] J. C. Willems, "Dissipative dynamical systems. I. General theory," Archive for Rational Mechanics and Analysis, vol. 45, pp. 321-351, 1972.

[2] D. J. Hill and P. J. Moylan, "The stability of nonlinear dissipative systems," IEEE Transactions on Automatic Control, vol. 21, no. 5, pp. 708-711, 1976. 
[3] D. J. Hill and P. J. Moylan, "Connections between finite-gain and asymptotic stability," IEEE Transactions on Automatic Control, vol. 25, no. 5, pp. 931-936, 1980.

[4] I. G. Polushin, A. L. Fradkov, and D. D. Khill, "Passivity and passification of nonlinear systems," Rossiǔskaya Akademiya Nauk. Avtomatika i Telemekhanika, no. 3, pp. 3-37, 2000.

[5] C. I. Byrnes, A. Isidori, and J. C. Willems, "Passivity, feedback equivalence, and the global stabilization of minimum phase nonlinear systems," IEEE Transactions on Automatic Control, vol. 36, no. 11, pp. 1218-1240, 1991.

[6] C. I. Byrnes and A. Isidori, "Asymptotic stabilization of minimum phase nonlinear systems," IEEE Transactions on Automatic Control, vol. 36, no. 10, pp. 1122-1137, 1991.

[7] A. J. van der Schaft, $L_{2}$-Gain and Passivity Techniques in Nonlinear Control, Springer, New York, NY, USA, 2000.

[8] W. Lin and T. Shen, "Robust passivity and feedback design for minimum-phase nonlinear systems with structural uncertainty," Automatica, vol. 35, no. 1, pp. 35-47, 1999.

[9] T. Shen, L. Xie, and K. Tamura, "Robust almost disturbance decoupling for nonlinear systems with structural uncertainty," in Proceedings of the 37th IEEE Conference on Decision and Control (CDC'98), pp. 4107-4108, Tampa, Fla, USA, December 1998.

[10] J. Yong and X. Y. Zhou, Stochastic control: Hamiltonian Systems and HJB Equations, Springer, New York, NY, USA, 1999.

[11] Z. G. Pan, "Canonical forms for stochastic nonlinear systems," Automatica, vol. 38, no. 8, pp. 1163-1170, 2002.

[12] P. Florchinger, "A passive system approach to feedback stabilization of nonlinear control stochastic systems," SIAM Journal on Control and Optimization, vol. 37, no. 6, pp. 1848-1864, 1999.

[13] P. Florchinger, "Stabilization of passive nonlinear stochastic differential systems by bounded feedback," Stochastic Analysis and Applications, vol. 21, no. 6, pp. 1255-1282, 2003.

[14] Z. G. Yan, G. S. Zhang, and J. K. Wang, "Non-fragile robust finite-time $H_{\infty}$ control for nonlinear stochastic Ito systems using neural network," International Journal of Control, Automation and Systems, vol. 10, no. 5, pp. 873-882, 2012.

[15] W. H. Zhang and B. S. Chen, "State feedback $H_{\infty}$ control for a class of nonlinear stochastic systems," SIAM Journal on Control and Optimization, vol. 44, no. 6, pp. 1973-1991, 2006.

[16] W. H. Zhang, H. S. Zhang, and B. S. Chen, "Generalized Lyapunov equation approach to state-dependent stochastic stabilization/detectability criterion," IEEE Transactions on Automatic Control, vol. 53, no. 7, pp. 1630-1642, 2008.

[17] W. H. Zhang and L. H. Xie, "Interval stability and stabilization of linear stochastic systems," IEEE Transactions on Automatic Control, vol. 54, no. 4, pp. 810-815, 2009.

[18] Z. W. Lin, J. Z. Liu, W. H. Zhang, and Y. G. Niu, "Stabilization of interconnected nonlinear stochastic Markovian jump systems via dissipativity approach," Automatica, vol. 47, no. 12, pp. 27962800, 2011.

[19] Z. W. Lin, J. Z. Liu, and Y. G. Niu, "Dissipative control of nonlinear stochastic systems with Poisson jumps and Markovian switchings," IET Control Theory and Applications, vol. 6, no. 15, pp. 2367-2374, 2012.

[20] R. Z. Has'minskii, Stochastic Stability of Differential Equation, Sijthoff \& Noordhoff, Alphen aan den Rijn, The Netherlands, 1980.

[21] H. J. Kushner, "Stochastic stability," in Stability of Stochastic Dynamical Systems, R. Curtain, Ed., vol. 294 of Lecture Notes in Mathematics, pp. 97-124, Springer, Berlin, Germany, 1972. 


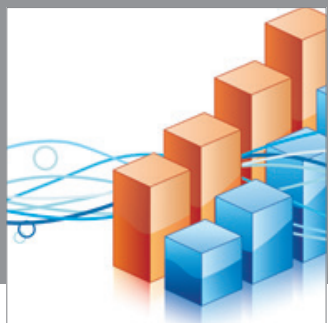

Advances in

Operations Research

mansans

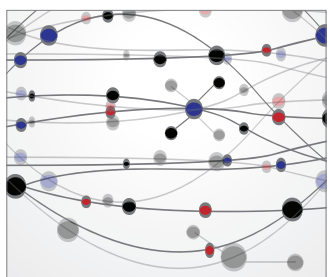

The Scientific World Journal
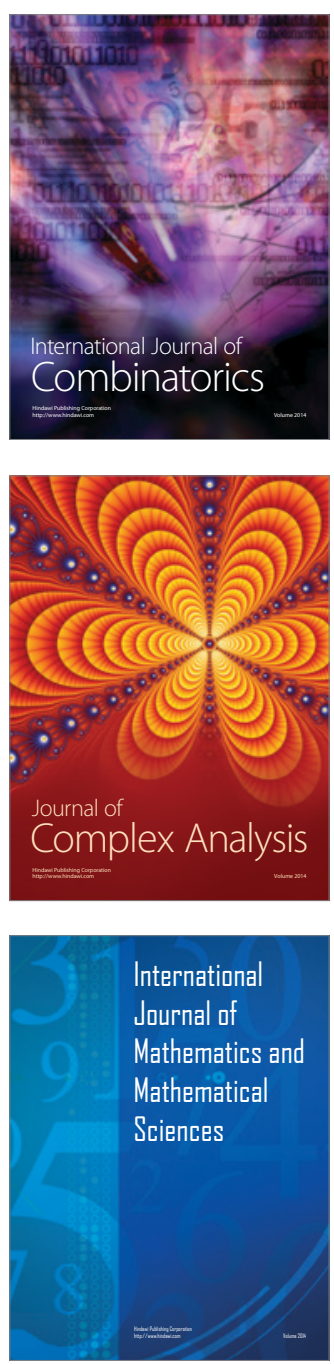
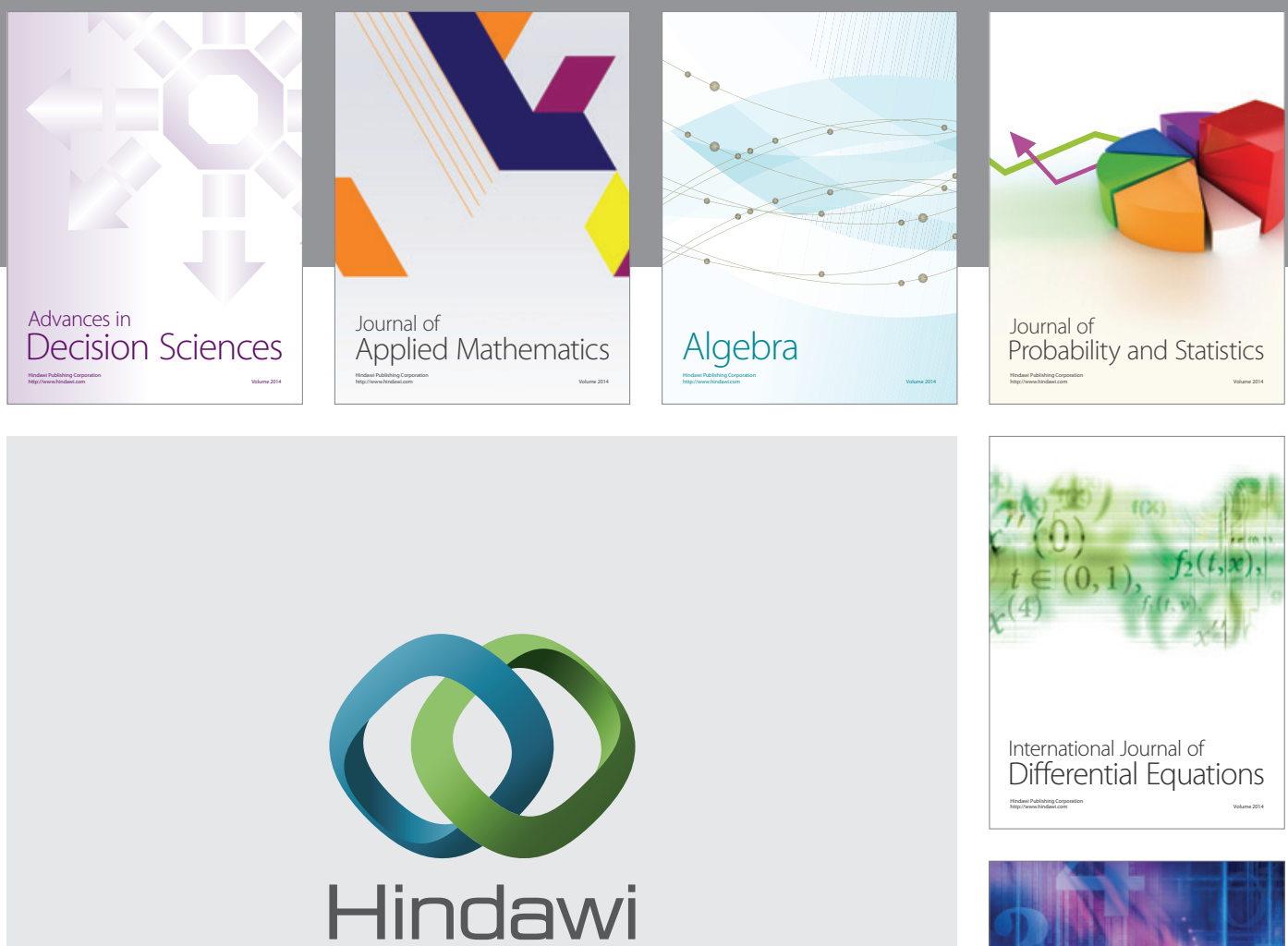

Submit your manuscripts at http://www.hindawi.com
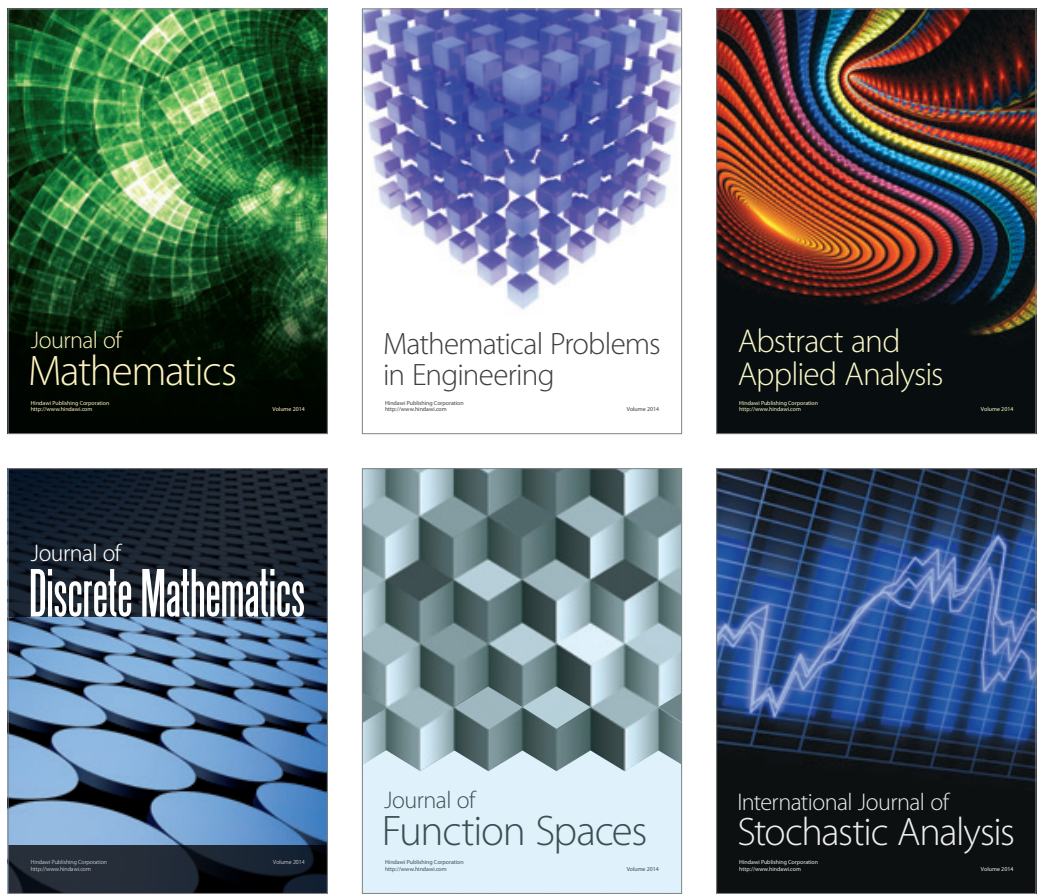

Journal of

Function Spaces

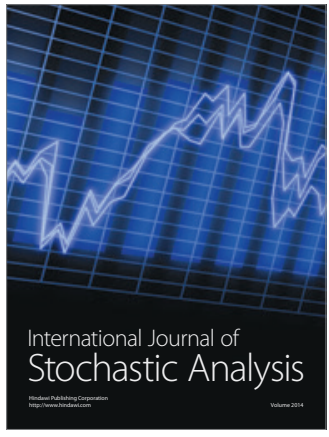

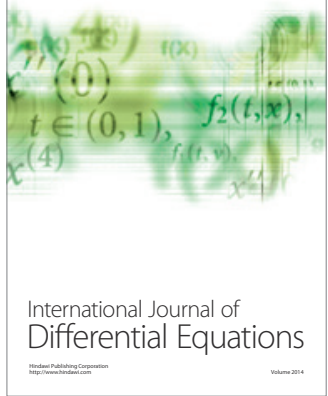
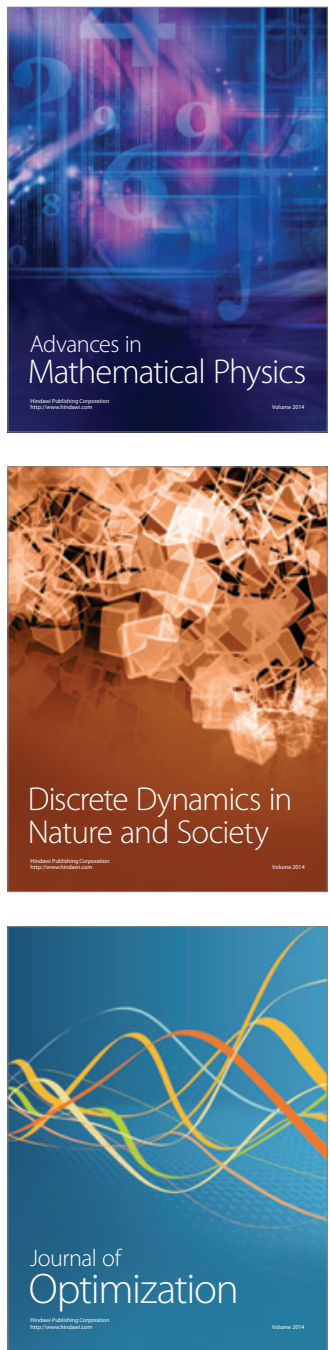